\title{
His Master's Voice
}

\section{Die soziale Konstruktion des ,Laien“ durch den ,Experten“}

Zusammenfassung: Dass Experten nicht einfach Personen(gruppen) sind, die im ,Besitz' eines spezifischen Wissens sind, welches den Laien ,fehlt‘, wurde in der Wissenschaftsforschung vielfach festgestellt. Expertise ist das Resultat eines komplexen diskursiven (und mithin auch historisch kontextualisierten) Prozesses, in dem unter anderem Verhandlungen von Deutungsansprüchen, semiotische Register, Sozialisationsprozesse, institutionelle Hierarchien und Wissensideologien eine zentrale Rolle spielen. $\mathrm{Zu}$ diesem Prozess gehört auch die Konstruktion und Abgrenzung von ,Laien“ durch ,Experten‘. Der Diskursfigur des ,Laien“ werden hierbei vielfach ex negativo Eigenschaften zu- bzw. abgeschrieben, die die Position der Expertin/des Experten legitimieren sollen (in der Sprachwissenschaft etwa, dass sie Sprache im Gegensatz zum ,deskriptiven“ Experten ,normativ‘ oder ,präskriptiv` betrachte). Prekär (für die Experten) wird diese Konstruktion immer dann, wenn die Laien nicht mitspielen, sich der Expertenschaft gegenüber also nicht als dankbar und lernbegierig, sondern als kritisch und skeptisch zeigen oder gar die Expertise in Frage stellen. Dieser Beitrag nimmt das komplexe Verhältnis von ,Experte‘ und ,Laie‘ in seiner diskursiven Bedingtheit und mit besonderer Berücksichtigung der Sprachwissenschaft in den Blick.

Abstract: As science studies have pointed out recurrently, experts are not simply (groups of) people who 'have' a specific knowledge which is 'lacking' to lay people. Expertise is the result of a complex discursive (and hence historically contextualized) process. Central to this process are negotiations of authority, specific semiotic registers, processes of socialization, institutional hierarchies, and ideologies of knowledge. Part of the process is the categorial distinction of 'lay people' from and by 'experts'. The 'lay' persona is thereby attributed with characteristics that relate, ex negativo, to the claimed position, competence, and authority of the expert (e. g., in linguistics, lay persons are constructed as having a 'normative' or 'prescriptive' relation to language, as opposed to the 'descriptive' linguist). This construct erodes, however, as soon as the lay persons

Spitzmüller, Jürgen: Institut für Sprachwissenschaft, Universität Wien, Sensengasse 3a, A-1090 Wien, juergen.spitzmueller@univie.ac.at 
refuse to take these attributed roles and challenge expertise. This chapter discusses this complex relationship of 'experts' and 'lays' in their discursive relativity. Linguistics serves as a case in point.

Schlüsselwörter: Expertise, Experte, Laie, Wissenschaftstheorie, Denkstil, Metapragmatik, soziale Positionierung

\section{Einleitung}

Die Gegenüberstellung von ,Laien“ und ,Experten', die dem vorliegenden Band (jedenfalls implizit) zugrunde liegt - sprachwissenschaftliche Expertinnen und Experten wurden eingeladen, über das Wissen von Laien über Sprache zu diskutieren - ist delikat. Was Laien sind und wie sie sich von ihrem Gegenpart, den Expert $^{\star}$ innen ${ }^{1}$, abgrenzen lassen, wurde in der Wissenschaftstheorie (vgl. Irwin \& Wynne 1998; Nieto-Galan 2016), der Soziologie (vgl. Z. Bauman 1987; Knorr Cetina 1999; Hitzler 1994; Schützeichel 2007; Pfadenhauer 2010), der Anthropologie (vgl. R. Bauman \& Briggs 2003; Carr 2010), der Sprachwissenschaft (vgl. Kalverkämper 1990; Antos 1996; Spitzmüller 2009; Bock \& Antos 2019; Hoffmeister 2019) und in andern Fächern vielfach diskutiert. In diesem Zusammenhang wurde immer wieder darauf hingewiesen, dass beides, Laie und Experte, 1. graduelle Konzepte sind (man ist nur mehr oder weniger Laie oder Experte), 2. Teil einer - in Anlehnung an Fleck (1999 [1935]: 53-54) ausgedrückt - ,dreigliedrigen` Beziehung, das heißt ergänzungsbedürftig: Man ist (mehr oder weniger) Laie in Bezug auf etwas, denn - wiederum mit Fleck (2011a [1936]: 296) ausgedrückt: „der Fachmann ist außerhalb seines Fachs - Laie“ (und man darf wohl ergänzen: Auch in Teilen seines Fachs trifft dies zu); Laie und Experte sind

1 Bezüglich der Genderformen sei angemerkt, dass in diesem Text, wie in der Literatur üblich, häufig von dem Experten und dem Laien im „typisierende[n] Singular“ (von Polenz 1988: 149) die Rede sein wird, der „,vorwiegend in androzentrischer maskuliner Form“ (von Polenz 1988: 149) auftritt und „auf dem Wege sich wiederholender textlicher Zuschreibungen geschichtsund sozialtypische sprachliche Bezugsklassen [schafft], also abstrakte Größen mit eigenem Realitätsstatus im Gegensatz zu demjenigen, was individuell-einmalig und unmittelbar real gelten kann“ (Lobenstein-Reichmann 2013: 81-82). Durch typisierende Singulare mit definitem Artikel gekennzeichnete Laien und Experten sind also stereotype Figuren (enactable personae sensu Goffman 1959), die in bestimmter Art und Weise sozial registriert (vgl. Spitzmüller 2013) sind. Dass dies im Maskulinum geschieht, ist dabei nicht unerheblich. 
außerdem 3. ko-konstitutiv: Laien gibt es nur, wo es Experten gibt, und umgekehrt.

Insbesondere der dritte Punkt soll im vorliegenden Beitrag etwas genauer beleuchtet werden. Der Beitrag wird sich also mit der Frage auseinandersetzen, inwiefern und zu welchem Zweck Laien und Experten sich gegenseitig konstituieren. Vor allem wird es dabei um die Frage gehen, wie und zu welchem Zweck Laien durch Experten diskursiv konstituiert werden. Damit ist auch zentral die Frage verbunden, welche Rolle ,der Laie، für das Selbstverständnis ,des Experten' spielt.

Hierfür diskutiert der Beitrag zunächst auf der Basis einschlägiger Arbeiten, warum ,Laie‘ und ,Experte“ konzeptionell aufeinander angewiesen, warum also ohne Laien keine Experten denkbar sind und umgekehrt. Dabei wird insbesondere gezeigt, warum die Figur des ,Laien“ für das Selbstverständnis des ,Experten' so wichtig ist und wie jene entsprechend kontrastiv zu diesem entworfen wird (Abschnitt 2). Anschließend wird Expertise als diskursive und soziale Praxis (meta-)pragmatisch in den Blick genommen (Abschnitt 3). Im Anschluss an diese Überlegungen diskutiert der Beitrag mit Blick auf die ,Laienlinguistik‘ (im Sinne einer Linguistik des sprachlichen ,Laienwissens'; vgl. Fingerhuth \& Boas 2018: 23) mögliche Relationssetzungen der Sprachwissenschaft $\mathrm{zu}$ ihrer ,Außenwelt‘ (Abschnitt 4).

\section{Zur bilateralen Konstruktion von ,Laie und ,Experte“}

\subsection{Der Laie als begehrter Begehrer}

Laie und Experte sind, wie vielfach herausgestellt wurde, ,als Gegensatzpaar konzipiert“ (Huber 2014: 24), das in intrikater Weise aufeinander verwiesen ist. Expert*innen versprechen Zugang zu etwas, was Laien ,begehren“, was diesen selbst aber nicht unmittelbar zur Verfügung steht, nämlich eine bestimmte Art von (synoptischem²) Wissen in einem bestimmten Gebiet:

2 Jedenfalls ist dies nach der wissenssoziologischen Definition von Hitzler (1994: 25-26) begriffsrelevant, da Hitzler die Figur des Experten von der des Spezialisten unterscheidet, der über Spezialkenntnisse zur Durchführung spezifischer Tätigkeiten verfügt, während dem Experten Überblickswissen zugeschrieben wird. 
Der Experte verfügt anscheinend über einen ausgesonderten Wissensbestand, der dem Nicht-Experten - jedenfalls in seiner Gesamtheit - nicht (ohne weiteres) zugänglich ist, der von diesem aber nachgefragt wird, auf den sich dieser im Hinblick auf bestimmte (und symptomatischer: auf immer mehr) lebenspraktisch relevante Fragen ver- und angewiesen sieht (bzw. glaubt). Der Experte wird vom Laien typischerweise konsultiert.

(Hitzler 1994: 26; Herv. i. Orig.)

Umgekehrt aber werden Expert`innen erst und nur durch „Anrufung“ (Althusser 1977 [1970]: 142) von Laien zu Expert*innen, und sie bleiben dies nur so lange, wie diese Anrufung Bestand hat:

Akteure werden zu Expert^innen - und sind es folglich nicht ein für alle Mal -, wenn andere Akteure ihnen bestimmte Problemlösungskapazitäten, über die sie selbst nicht verfügen, unterstellen, diese nachfragen und/oder aktiv ,konsumieren'.

(Antony \& Pfadenhauer 2019: 2)

Das heißt, dass nicht nur Laien Expertinnen (zur Lösung bestimmter Problemstellungen) benötigen, sondern dass auch Expertinnen Laien benötigen, die ihnen ihren Expert ${ }^{\star}$ innenstatus überhaupt erst zusprechen bzw. ihrer Expertenfigur ein Auditorium geben. Daher sind Laien als ,begehrende Figuren' ihrerseits bei Expertinnen begehrt.

Daraus ergibt sich allerdings, dass man die Figur des Laien nicht einfach mit der des ,Nicht-Experten“ (die ja in den beiden wissenssoziologischen Zitaten als Gegensatzfigur genannt wird) gleichsetzen kann, denn Laien verbindet mit Expert`innen ein Bezug zu und in der Regel auch das Interesse an einem bestimmten Gegenstand. Laien sind somit nicht einfach nur Akteure, die etwas ,nicht (hinreichend) wissen', sondern solche, die etwas ,(genauer) wissen wollen':

Allgemein wird man wohl sagen können, dass in der funktional differenzierten Gesellschaft ,Laie، die Bezeichnung für eine Position ist, in welcher man als Abnehmer oder Nachfrager spezifischer von ,Experten“ angebotener Leistungen in den Kommunikationszusammenhang der Subsysteme eintritt.

(Schützeichel 2007: 547)

Nicht jeder ,Nicht-Experte، ist somit auch Laie, denn es gibt ja durchaus - und in vielen Gebieten überwiegend - Nicht-Experten, die an bestimmten ExpertenAngeboten keinen Bedarf, für das betreffende Phänomen kein Interesse oder vielleicht nicht einmal Kenntnis von dessen Existenz haben (viele von uns sind Nicht-Experten im Schwingsport, was uns aber noch lange nicht zu Schwingsport-Laien macht). ,Laien` nämlich befassen sich - wie Expert`innen - mit einem bestimmten Thema, dies aber aus einer anderen sozialen Position her- 
aus, welche keineswegs durch Wissensdifferenzen bestimmt sein muss, sondern beispielsweise auch durch Differenzen in der (formal-institutionellen) Qualifikation oder der ökonomischen Einbindung (der Laie als ,,jemand [...], der nicht für seine Tätigkeit bezahlt wird“; Schützeichel 2007: 547) bestimmt sein kann. Das Gegensatzpaar Laie/Experte verweist somit immer auch auf soziale und kulturelle Zusammenhänge, innerhalb derer die entsprechenden Positionen verortet sind, und mithin auch auf skalierbare Formen von Macht, nicht zuletzt Handlungs- und Kommunikationsmacht bzw., soziolinguistisch ausgedrückt, agency (Duranti 2006) und voice (Blommaert 2005: 68).

\subsection{Ex-negativo-Konstruktionen}

Dass die Gegensatzfigur dabei alles andere als symmetrisch konzipiert ist, zeigt Kalverkämper auf der Basis etymologischer und lexikographischer Analysen:

Die sprach- und kulturgeschichtlichen Tatsachen belegen es [...]: Der Laie definiert sich negativ zum Fachmann; es ist nicht umgekehrt; vielmehr sind begrifflich der Fachmann und sein fachliches Können der Maßstab, an dem sich der Begriff des ,Laien“ orientiert, wenn er - in negativer Bestimmung - mit ,Nichtfachmann' synonym gebraucht wird, wenn die Wörterbücher für ,Laie‘ angeben ,ungelehrt', ,etwas nicht gelernt habend‘, ,von etwas nichts verstehend', ,unvertraut mit einer Sache', ,nicht versiert', ,ohne Kenntnis‘.

(Kalverkämper 1990: 97; Herv. i. Orig.)

Der Begriff des ,Fachmanns‘ entwickelt sich, wie Kalverkämper ausführt, aus der ursprünglichen Bedeutung von ahd. fah , abgrenzen, eingrenzen` zuerst; der ,Laie' wird erst in einem zweiten Schritt - zunächst bekanntlich im klerikalen Bereich als Bezeichnung für Nicht-Kleriker - und ex negativo zum ,Fachmann“ gebildet. $^{3}$ Kalverkämper selbst lehnt diese binäre Opposition im Weiteren ab und schlägt vor, stattdessen von einem Kontinuum zwischen ,Laie“ und ,Fachmann' auszugehen, das durch zu- und abnehmende ,Fachlichkeit‘ konstituiert werde. In seiner merkmalsemantischen Diktion klingt das so:

Demnach wäre dann deren Beziehung geprägt von einer verbindenden Eigenschaft, nämlich dem Fachlichen, der Qualität der ,Fachlichkeit‘. Die Kategorie ,Fachmann‘ ist dazu merkmalhaltig - oder sinnvoller gesagt: merkmalreich [...]; und die zum ,Fachmann` oppositive, negative Kategorie ,Laie‘ ist dazu merkmalarm oder besser: merkmalreduziert.

(Kalverkämper 1990: 98; Herv. i. Orig.)

3 Experte wird Anfang des 19. Jahrhunderts aus dem Französischen entlehnt und geht auf lat. expertus (,erfahren, kundig, erprobt, bewährt') zurück (vgl. Strauß et al. 2011: 504; Huber 2014: 24). 
Kalverkämper ist überzeugt, auf diese Weise die vor allem für den Laien degradierende Polarität aufheben zu können, indem eben das ,Gemeinsame‘ betont wird. Dass auch hier Laien vor allem über ein Defizit - das relative Fehlen von ,Fachlichkeit“ - definiert werden, ist allerdings durchaus bemerkenswert.

\section{Laientheorien}

Implizit/nicht formalisiert

Inkohärent/inkonsistent

Verifikationsorientiert

Unkontrollierte Kausalitäten

Beschreibend

Intrinsische Pragmatik

Spezifisch

Schwache Evidenz
Expertentheorien

Explizit/formalisiert

Kohärent/konsistent

Falsifikationsorientiert

Kontrollierte Kausalitäten

Erklärend

Extrinsische Pragmatik

Generalisierbar

Starke Evidenz

Abb. 1: ,Laientheorien“ vs. ,Expertentheorien“ (nach Furnham 1988: 2-7; vgl. Bock \& Antos 2019: 57-58).

Andere Versuche, Laien und Experten differenzierter voneinander abzugrenzen, sind in dieser Hinsicht nicht besser. Das gilt etwa für solche Ansätze, die versuchen, Laien und Experten über die Art und Weise, wie sie Wissen bzw. Theorien generieren und prozessieren, $\mathrm{zu}$ differenzieren. Der Psychologe Adrian Furnham (1988: 2-7) etwa nennt acht Unterscheidungskriterien, die sich aus bestimmten Wissenschaftsidealen ableiten (vgl. Abbildung 1), nämlich der Vorstellung, wissenschaftliche Theorien haben expliziert und formalisiert, kohärent, falsifikationsorientiert, kontrolliert in der Beschreibung von Kausalitäten, erklärend, auf externe Handlungsfaktoren achtend, generalisierbar und empirisch stark zu sein - was nun alles für Laientheorien gerade nicht zutreffe. ${ }^{4}$ Interessant an dieser Aufstellung ist nicht nur, dass ,Laientheorien“ auch hier im Wesentlichen ex negativo als defizitäre (,unwissenschaftliche`) Theorien konzipiert werden, sondern auch, dass die Kriterien, die hier für die ,Wissenschaftlichkeit“ von Theorien angeführt werden, alles andere als unkontrovers sind. Jedenfalls zielen sie auf ein sehr spezifisches (empiristisches, kausalistisches und nomo-

4 Vgl. hierzu auch Bock \& Antos (2019: 57-58). 
thetisches, eher naturwissenschaftliches ${ }^{5}$ ) Wissenschaftsverständnis, das keinesfalls mit ,Wissenschaftlichkeit“ generell gleichgesetzt werden kann. Das sieht man auch daran, dass einige der genannten Faktoren auch zur wissenschaftsinternen Differenzierung (etwa zwischen empiristischen und rationalistischen oder zwischen quantitativen und qualitativen Ansätzen) herangezogen werden, und dass sie vielfach, wie die soziologische Wissenschaftstheorie in der Nachfolge von Fleck (1999 [1935]) und Kuhn (1999 [1962]) gezeigt hat (vgl. Longino 2016), eher einem Wunschdenken als der Realität entsprechen (und übrigens auch ineffektiv wären ${ }^{6}$ ). Bemerkenswert ist zudem, dass die von Furnham den ,Laientheorien“ zugeordnete Seite der Skalen häufig in wissenschaftsinternen Streitigkeiten zur Disqualifikation von Zugängen verwendet wird, im akademischen Diskurs also offensichtlich stark deontisch funktional ist. ${ }^{7}$ Außerdem fällt auf, dass die Kriterien sich stark mit den Merkmalen decken, die Zygmunt Bauman (1987) als charakteristisch für ,moderne‘ (im Gegensatz zu ,postmodernen') Wissensideologien ausgemacht hat:

The typically modern view of the world is one of an essentially orderly totality [...]. Effectivity in control and correctness of knowledge are tightly related (the second explains the first, the first corroborates the second), whether in laboratory experiment or societal practice. Between themselves, they supply criteria to classify existing practices as superior or inferior. [...] Practices which cannot be objectively justified [...] are inferior as they distort knowledge and limit effectivity of control. Moving up the hierarchy of practices measured

5 Vgl. Coupland (2001); Joas \& Knöbl (2004: 13-38).

6 Wie Longino (2016) herausstellt, beruht der Großteil wissenschaftlicher Erkenntnis nicht auf Falsifikation und Empirie, sondern auf Vertrauen (mit Russell 1910-1911: auf knowledge by description), da ein Replizieren und Nachprüfen äußerst ineffektiv wäre. Ineffektiv - und nach Auffassung von Wissenschaftstheoretikern, die die Sinneswahrnehmung für trügerisch oder jedenfalls unterspezifiziert halten (vgl. Quine 1953 [1951] und im Anschluss daran Chomsky 2002: 98-99) auch epistemologisch fatal - wäre auch die Aufgabe einer Theorie nach Falsifikation im Sinne Poppers (2005 [1935]), die Fleck (1999 [1935]: 40) und Kuhn (1999 [1962]) zufolge ohnehin die Ausnahme ist, da Theorien (und Theoretiker*innen) sich als notorisch resistent gegen Widersprüche und Widerlegungsversuche erweisen, was man mit Blick auf die Kohärenz der Wissenschaftsgeschichte durchaus positiv bewerten kann: Gäben die Theoretiker jedem empirisch gerechtfertigten Widerstand statt, wäre diese eine Geschichte voller Rupturen und radikalen Wendungen, was jedenfalls die Konstruktion einer konsistenten Forschungs- und Forscherbiographie sehr erschweren würde (die ohnehin auf Glättungen und Löschungen widersprechender Fachentwicklungen beruht; vgl. Kuhn 1999 [1962]: 148).

7 Ein sehr plastisches sprachwissenschaftliches Beispiel hierfür ist die in die germanistischen (Wissenschafts-)Geschichtsbücher eingegangene Debatte zwischen Ludwig Jäger (1993a, 1993b) und Günther Grewendorf (1993), die im Wesentlichen die bis in die Antike zurückreichende Debatte zwischen Empiristen (Jäger) und Rationalisten (Grewendorf/Chomsky) repliziert. 
by the control/knowledge syndrome, means also moving toward universality and away from 'parochial', 'particularistic', 'localized' practices.

Die Politik der von Bauman so genannten modernen Intellektuellen bestehe nun darin, im Sinne eines ,Gesetzgebers‘ autoritative Äußerungen vorzunehmen, deren Autorität sich darauf berufe, dass diesen Personen im Gegensatz zu anderen ein besserer Zugang zum ,echten (objektiven) Wissen` offenstehe:

The typically modern strategy of intellectual work is one best characterized by the metaphor of the 'legislator' role. It consists of making authoritative statements which arbitrate in controversies of opinions and which select those opinions which, having been selected, become correct and binding. The authority to arbitrate is in this case selected by superior (objective) knowledge to which intellectuals have better access than the non-intellectual part of society.

(Z. Bauman 1987: 4)

Wie die Sprachanthropologen Richard Bauman und Charles Briggs in ihrem für das hier verhandelte Thema einschlägigen Buch Voices of Modernity (vgl. R. Bauman \& Briggs 2003: 127) in direktem Anschluss an diese Argumentation Zygmunt Baumans zu Recht betonen, geht dieser Selbstüberhöhung über die „non-intellectual Others“ aber ein Prozess voraus, in dem diese ,NichtIntellektuellen' als Gruppe überhaupt erst konstruiert werden (und den die Autoren im 17. und 18. Jahrhundert in Europa lokalisieren). Außerdem weisen Bauman und Briggs auf einen Punkt hin, über den später noch zu sprechen sein wird (vgl. Abschnitt 3): Diese Konstruktion und Grenzziehung schließt ganz wesentlich auch bestimmte kommunikative Praktiken und (Denk-)Stile ${ }^{8}$ ein:

[...] assuming authority over the non-intellectual Others, but also the prior task of constructing the non-intellectual Others [...] were oriented towards the construction of a metadiscursive regime of intellectual authority, constituted in terms of ways of speaking as well as ways of knowing.

(R. Bauman \& Briggs 2003: 127; Herv. i. Orig.)

Ähnlich expertenzentriert wie Furnhams Vorschlag ist auch die von Giddens (1987: 7) im Rahmen seines Konzepts der doppelten Hermeneutik vorgeschlagene Idee, Laienwissen sei ,empraktisch', Expertenwissen ,handlungsentbun-

8 Vgl. zum auch Kommunikation einschließenden Denkstilkonzept Flecks ausführlich Andersen et al. (2018). 
den'. ${ }^{9}$ Nur aus der handlungsentbundenen Vogelperspektive der Experten, so Giddens weiter, ließen sich sog. nicht-diskursive Zusammenhänge (wie Giddens die Strukturperspektive nennt) erkennen und benennen. Problematisch ist diese Unterscheidung dann, wenn sie (wie bei Giddens) impliziert, Laien seien zur Abstraktion aus ihrer Lebenswelt nicht oder nur sehr bedingt in der Lage und Experten seien in ihrem Expertenhandeln nicht oder nur sehr bedingt lebensweltlich gebunden. Ersteres schließt eine ganze Reihe von sozialen Akteuren aus, die handlungsentlastet über Lebenswelten (oder Sprache) reflektieren, aber nicht als Experten gelten (wollen oder können), letzteres ignoriert die vielfältigen sozialen und politischen Interessen, in die Expertenhandeln eingebettet ist (Giddens selbst mag dafür als Beispiel dienen).

\subsection{Expertise und/als Diskursmacht}

Aber vielleicht ist gerade dies der entscheidende Punkt: Die Frage, die Giddens umtreibt, ist, wozu die Welt Soziologen braucht, eine Berufsgruppe, deren zentrales Legitimationsproblem darin bestehe, dass sie - wie Giddens (1987: 8) zufolge übrigens auch Linguisten - letztlich nur das studierten, was alle betroffenen sozialen Akteure ohnehin wüssten (und sogar wissen müssten, wenn auch nur im Sinne eines impliziten Handlungswissens). Giddens' Antwort auf dieses ihn selbst betreffende Dilemma fällt ganz im Sinne des Bauman'schen ,modernen Intellektuellen' aus: ${ }^{10}$ Die Welt braucht Soziologen, weil diese einen Blick auf Gesellschaft haben, den sonst niemand habe - einen Blick von ,außen'. Und dieser exklusive Blick sei für die Erlangung von „good reasons“ wesentlich:

The main role of the social sciences in respect of the critique of common sense is the assessment of reasons as good reasons in terms of knowledge either simply unavailable to lay agents or constructed by them in a fashion different from that formulated in the metalanguages of social theory.

(Giddens 1984: 339)

Dies führt uns zu einem wesentlichen Aspekt von Expertise: ihre Beanspruchung im Sinne eines Anspruchs auf Deutungshoheit und mithin Diskursmacht. Dieser Akt der Beanspruchung erfordert nun aber der oben ausgeführten Argu-

9 In der Linguistik wird eine ähnliche Idee unter anderem von Paul (1999: 194) vertreten (und mit Verweis auf Paul dann auch von Spitzmüller 2009 propagiert); allerdings geht Paul nicht von Giddens, sondern unter anderem von Garfinkel aus (auf den sich seinerseits auch Giddens beruft).

10 Vgl. dazu auch kritisch Kim (2004). 
mentation von Bauman und Briggs zufolge die diskursive Konstruktion des ,Laien“ als Figur der Außenwelt eigener Expertise. Expertenarbeit ist somit, wie Bock \& Antos (2019: 64) unter Verweis auf Bogner (2005) festhalten, mit „Grenzpolitik“ verbunden - einer Politik, in der es „nicht unwesentlich“ darum gehe, „Nichtwissen und Ungewissheit zu erkennen und in Expertisen in Rechnung zu stellen“ und die durch ihre schiere Notwendigkeit „die Brüchigkeit der scheinbar so geläufigen Dichotomisierung von ,Experten“ und ,Laien““ gerade unterstreiche. ${ }^{11}$

Das bedeutet nun natürlich nicht, dass Experten- (und ex negativo Laien-) zuschreibungen nicht auf einer ganzen Reihe von Bedingungen beruhen, etwa bestimmten technischen Fertigkeiten, institutionellen Kompetenzen, Positionen, Zertifikaten usw. Denn freilich,

[...] un docteur en médecine qui commet une série de fautes professionnelles et un architecte qui ne réussit pas à construire une seule maison stable et fonctionnelle perdront assez vite leur image d'experts. D'autre part, une personne même lorsqu'elle est capable de présenter des thèses intéressantes, disons par exemple en biochimie, dans la plupart des cas, ne sera considérée comme « expert » ni par la communauté scientifique ni par la société en général sans avoir passé les différentes phases de formation secondaire et universitaire (bac, licence, maîtrise, doctorat ...). ${ }^{12}$

(Stegu i. Dr.)

Experten werden also an ihren feldspezifischen Beiträgen und deren (institutioneller wie anlassspezifischer) Angemessenheit ,gemessen'. Gerade dabei spielen aber auch diskursive Maßstäbe eine wesentliche Rolle. Nicht nur, ob das Haus ,stabil' und ,funktional' ist, ist wichtig, sondern auch, was gesellschaftlich als ,stabiles' und ,funktionales' Haus gilt. ${ }^{13}$ In diesem Zusammenhang wird

11 Die beschriebene „Grenzpolitik“ ist natürlich keine, die nur die Grenze zwischen Innenund Außenwelt der Wissenschaft betrifft, sondern auch Wissenschaftslandschaften parzelliert. Das Argument des ,superioren Blicks‘ etwa wird ja auch in binnenwissenschaftlicher Grenzarbeit verwendet und hat, wie Agha (2007) herausgearbeitet hat, in der Herausbildung der Linguistik als Fach und ihrer Abgrenzung von anderen Disziplinen, die sich schon längst handlungsentlastet mit Sprache befasst haben, eine wesentliche Rolle gespielt.

12 Übers. J. S.: „Ein Arzt, der eine Reihe von Kunstfehlern begeht und ein Architekt, der nicht in der Lage ist, ein stabiles und funktionales Haus zu entwerfen, werden sehr bald ihre Expertenzuschreibung los sein. Andererseits aber kann jemand (zum Beispiel in der Biochemie) noch so interessante Thesen präsentieren; ohne entsprechende formale Qualifikation (Bakkalaureat, Lizenziat, Magister, Doktorat ...) wird diese Person meistens trotzdem nicht als ,Experte‘ akzeptiert, weder in der Wissenschaft noch in der Gesellschaft generell.“

13 Das gilt, wie Fleck (1999 [1935]) vielfach gezeigt hat, für den Mediziner und die Frage, wie man Krankheit und Gesundheit definiert, nicht weniger. Vgl. dazu auch Bogner (2005), der den 
relevant, was Foucault (1997 [1971]: 22) die Disziplinen genannt hat, „einen Bereich von Gegenständen, ein Bündel von Methoden, ein Korpus von als wahr angesehenen Sätzen, ein Spiel von Regeln und Definitionen, von Techniken und Instrumenten“, und das, was Fleck (1999 [1935]: 130) als Denkstilgebundenheit bezeichnet,

gerichtetes Wahrnehmen, mit entsprechendem gedanklichen und sachlichen Verarbeiten des Wahrgenommenen [...] [, charakterisiert durch] gemeinsame Merkmale der Probleme, die ein Denkkollektiv interessieren; der Urteile, die es als evident betrachtet; der Methoden, die es als Erkenntnismittel anwendet. Ihn begleitet eventuell ein technischer und literarischer Stil des Wissenssystems.

(Fleck 1999 [1935]: 130; Herv. i. Orig.)

Erfolgreiche Expertenarbeit erfordert also auch eine glaubhafte Demonstration der Kompatibilität eigenen Expertenhandelns mit disziplinär akzeptierten und validierten Methoden, Arbeitspraktiken, aber auch Argumentationsstilen. All dies ist Teil der Expertenperformance, von der im folgenden Abschnitt die Rede sein wird.

\section{Expertenperformance und die Metapragmatik der Expertise}

[...] expertise is not something one has but something one does [...].

(Carr 2010: 17)

Betrachtet man Expertise, wie es die Sprachanthropologin Summerson Carr im Rahmen eines detaillierten Literaturberichts $\mathrm{zu}$ vor allem anthropologischer Literatur zum Thema vorschlägt, nicht als ,Eigenschaft‘ oder ,Besitz‘, sondern als diskursive Praktik - etwas, was „zur Aufführung gebracht“ wird -, so ergeben sich daraus für das Verständnis des Verhältnisses von Laien und Experten wertvolle Erkenntnisse. Dies soll im Folgenden gezeigt werden. Dazu ist es nötig, Carrs Konzeption von Expertise als performativem Phänomen etwas genauer vorzustellen.

Carrs Vorschlag zufolge ist Expertise als inhärent interaktionales und interpretatives Phänomen zu betrachten. Außerdem sei sie notwendigermaßen ideo-

Umgang von Expert^innen mit kategorialen Unsicherheiten im Bereich der Pränataldiagnostik analysiert. 
logisch (,inescapably ideological“), da die Legitimation von Expertenwissen auf komplexen Werthierarchien beruhe und immer auch mit Macht(ansprüchen) in Verbindung stehe (Carr 2010: 17). Expertise ist dabei, wie Carr mit Verweis auf zahlreiche anthropologische und soziologische Untersuchungen zum Thema ausführt, an bestimmte (institutionell ritualisierte) Formen der Performanz gekoppelt, an Expertisenrituale wie Experteninterviews, Gutachten, PeerReview-Verfahren oder auch so komplexe kommunikative Prozeduren wie Tagungen (wie jene, aus welcher der vorliegende Band - als weitere Expertisenperformance - hervorgegangen ist). Vom Warming-up über die Vorträge und Diskussionen bis hin zu den Kaffeepausengesprächen und Conference Dinners: überall wird dabei (auch) Expertise zelebriert und verhandelt. Dabei spielt, wie Carr betont, auch die sprachliche Darstellung eine zentrale Rolle.

After all, to be an expert is not only to be authorized by an institutionalized domain of knowledge or to make determinations about what is true, valid, or valuable within that domain; expertise is also the ability to "finesse reality and animate evidence through mastery of verbal performance” (Matoesian 1999, p. 518).

(Carr 2010: 19)

Wer sich an ihre/seine ersten Tagungsteilnahmen erinnert, kann sich sehr wahrscheinlich leicht wieder ins Bewusstsein rufen, dass das Verhalten in solchen ritualisierten Expertenperformances nichts ist, was man einfach so ,kann'. Expertise muss also (auch in performativer Hinsicht) erlernt werden, sie beruht, wie auch Carr (2010: 19-21) betont, auf Sozialisation und Nachahmung.

Bereits Fleck hat darauf hingewiesen, dass Experte zu werden bedeutet, bestimmte Sichtweisen, aber auch körperliche Praktiken (wie das richtige SichBeugen über und Schauen in ein Mikroskop in Flecks eigenem Fach, der Bakteriologie $^{14}$ ) zu lernen und andere loszuwerden (Fleck 1999 [1935]: 121 spricht von „gerichtete[m] Wahrnehmen“):

Der so gefaßte Denkstil ist das Ergebnis theoretischer und praktischer Ausbildung der gegebenen Person, und indem er vom Lehrer auf den Schüler übergeht, stellt er einen gewissen traditionellen Wert dar, der einer spezifischen geschichtlichen Entwicklung und spezifischen soziologischen Gesetzen unterliegt.

(Fleck 2011b [1935]: 219)

Erworben werden muss, wie Carr betont, auch das entsprechende Register. ${ }^{15}$ Dieses dient nicht zuletzt auch der Grenzarbeit zwischen Experten und Laien,

14 Vgl. Fleck (2011c [1935]: 211-212); dazu ausführlicher Andersen et al. (2018: 31-36).

15 Zum sprachanthropologischen Registerbegriff vgl. Spitzmüller (2013). 
da es die Kommunikationsakteure metapragmatisch gegenüber Laien als Experten markiert und positioniert. Expertensprechen ist mithin nicht einfach ein (sozial profiliertes) Sprechen über ein bestimmtes Fachgebiet, sondern das Sprechen über dieses Fachgebiet in einer bestimmten Art und Weise und zu einem bestimmten Publikum:

[...] people emerge as more or less expert not in unmediated relationships to culturally valued objects (such as wine), but instead through the discursive processes of representing them (such as wine talk). [...] The enactment of expertise may involve talking to even more than it entails talking about.

(Carr 2010: 23; Herv. J. S.)

Des Weiteren ist Expertise verbunden mit Autorisierungs- und Legitimierungspraktiken, in denen es vor allem darum geht, eine Vertrautheit mit bestimmten Objekten oder Phänomenen (Hirnscans, Wein, die Geschichte einer Sprache) glaubhaft darzustellen - idealerweise, wie Knorr Cetina in ihrer Expertendefinition hervorhebt, so glaubhaft, dass man damit auch das Vertrauen anderer Expert*innen erwirbt, die keinen oder weniger Zugriff auf diese Objekte haben:

Experts are those who have learned to engage with objects in reliable trust relationships and who, therefore, are trusted by colleagues who cannot engage in those relationships directly.

(Knorr Cetina 1999: 135)

Falls es sich um weithin zugängliche Phänomene handelt, wie dies in (jedenfalls gegenwarts- und auf das direkte Umfeld der beteiligten Akteure bezogenen) Formen der Sozial- und Kulturwissenschaften häufig der Fall ist, werden in Expertisepraktiken ,relative Lesbarkeiten“ (Carr 2010: 22) dieser Phänomene konstruiert. Beispiele hierfür sind die esoterische Geschmackslexik und die Verkostungsrituale, deren sich Wein-Connaisseure bedienen, um ihre sensorische Überlegenheit zu demonstrieren (vgl. Silverstein 2003: 222-227), die von Giddens beanspruchte ,superiore Außenperspektive‘ der Soziologie auf Gesellschaft, aber auch der vielfach und laut propagierte (Alleinvertretungs-)Anspruch der sog. modernen Sprachwissenschaft darauf, Sprachgebrauch und Sprachwandel ,objektiv‘ und damit ,richtig' beschreiben zu können (vgl. bspw. Hall 1950; Lyons 1968: 2; Sanders 1998; Denkler 2008; Meinunger 2008; Anderwald 2012). ${ }^{16}$

Zusammenfassend lässt sich die Argumentation dieses Abschnitts wie folgt formulieren: Expertise wird in komplexen, kommunikativ konstituierten und in

16 Vgl. dazu ausführlich Cameron (1995), Spitzmüller (2005a) und Spitzmüller (2019). 
einer bestimmten Art und Weise mediierten Positionierungspraktiken generiert, in denen sich Akteure selbst als Expertinnen darstellen, darstellen wollen oder dargestellt werden. Dies funktioniert aber nur in Ausrichtung zu und Abgrenzung von anderen Akteuren: den Mit-Expert*innen und den Laien. Erst in diesem polyphonen Chor erscheinen bestimmte Stimmen dann als Stimmen von Meistern.

\section{His Master's Voice und Mastered Voices}

Wie lässt sich nun auf der Grundlage solcher Überlegungen zur KoKonstruktivität von Experte und Laie das (ja zyklisch immer wieder und in Relation zur parallelen Formalisierung und sozialen Abstrahierung der Linguistik auftretende ${ }^{17}$ ) Interesse von Teilen der Sprachwissenschaft an ,Laien“ bewerten? Dazu ist zunächst einmal zu diskutieren, welche Rolle den Laien in der ,laienorientierten' Forschung überhaupt zugeschrieben wird. Lange Zeit war es ja (und in Teilen der Linguistik ist es immer noch) so, dass das sprachwissenschaftliche Idealbild des ,Laien“ dem Jack-Russell-Terrier Nipper glich, der andächtig, aufmerksam und ehrfürchtig der Stimme seines Herrn ${ }^{18}$ lauscht (Abbildung 2):

[...] the message that linguistics has for our society at present is primarily the one that we have used as the title of this book: LEAVE YOUR LANGUAGE ALONE! [...] this message is both negative and positive. It is negative, in that it warns us to give up, to abandon entirely the old dogmatic, normative, theological approach of traditional grammar and of social snobbery; and to substitute the relativistic, objective approach of scientific study and analysis. It is positive, in that it tells us, once we've cleared the ground in this way, to go ahead and to find out for ourselves what the facts really are, to analyze and describe them as accurately as we can, and then to apply the knowledge we have obtained in that way. In both these respects, the contribution of linguistics is simply a part of the effort of all science in modern democratic society, to find out the truth and to act upon it; in this sense, the linguistician, like other scientists, may take as his motto the noblest of all slogans: "Ya shall know the truth, and the truth shall make you free."

(Hall 1950: 248-249)

17 Vgl. dazu ausführlich Knobloch (2005).

18 Zur Geschichte des ursprünglich Dog looking at and listening to a Phonograph betitelten Bildes des britischen Malers Francis Barraud und der Bildmarke His Master's Voice vgl. bspw. Suisman (2009: 111- 121). 


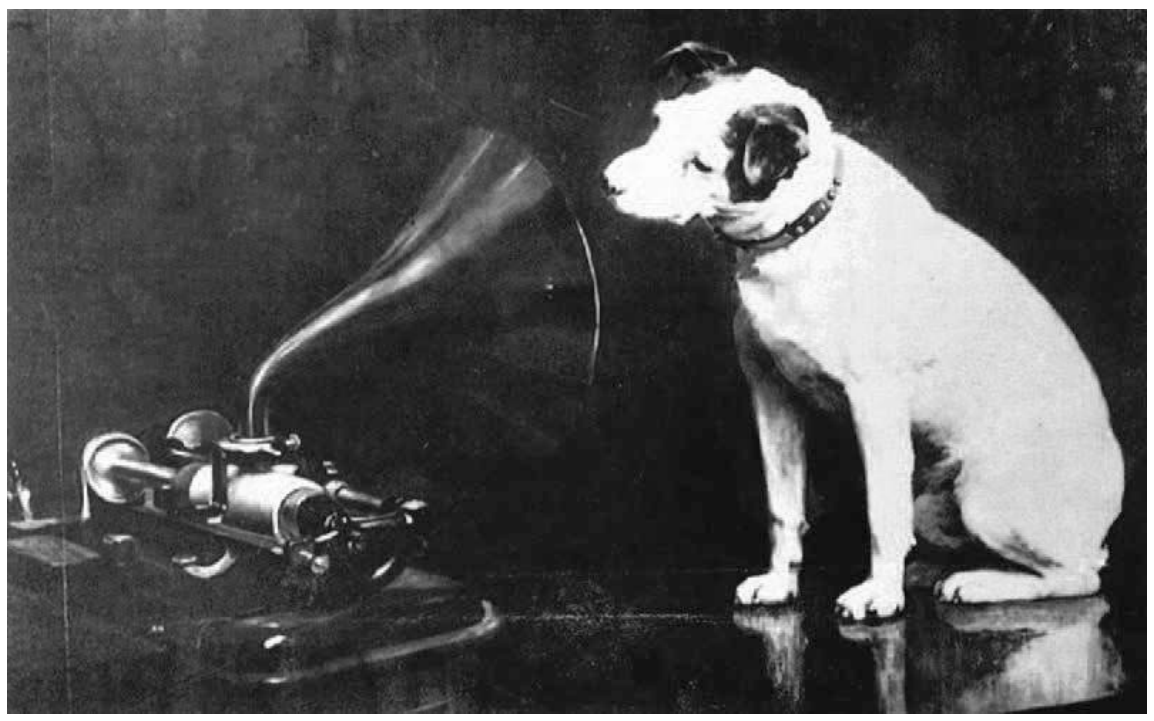

Abb. 2: Francis J. Barraud: His Master's Voice (1898)

Quelle: https://upload.wikimedia.org/wikipedia/commons/b/bd/OriginalNipper.jpg (letzter Zugriff: 17.02.2021).

Hier werden Laien ganz offensichtlich zu (mit ,Weisheit‘ bezahlten) Claqueuren degradiert, deren einzige Aufgabe es ist, die Brillanz der Expertinnen zu bejubeln (oder aber wenigstens dankbar für die geleistete Aufklärung zu sein). Sie dienen als Ex-negativo-Figuren der Selbstvergewisserung der sprachwissenschaftlichen professionellen Identität (vgl. Cameron 1995: 5). Dass sich Laien im oben ausgeführten Sinn also Akteure, die sich auch für Sprache interessieren, dies aber aus einer anderen sozialen Position heraus tun als Sprachforscher ${ }^{\star}$ innen $^{19}$ - mit dieser Rolle nicht zufrieden geben wollen, hat die anhalten-

19 Sprachforschung bezeichnet hier im Anschluss an Maas (2016: 1) „ein wissenschaftliches Feld [...], das nicht durch disziplinäre Schranken eingeschränkt ist“. Damit wird der Tatsache Rechnung getragen, dass sich das hier Ausgeführte nicht nur auf die disziplinäre Linguistik bezieht, sondern auch auf andere mit Sprache befasste Disziplinen wie die Sprachanthropologie, Sprachsoziologie, Sprachpsychologie usw. Das ist auch deshalb wichtig, weil die zentralen Konzepte und Argumente, die expertenseitig im hier besprochenen Diskurs ins Feld geführt werden, in aller Regel gar nicht aus der Linguistik im engeren Sinne stammen, sondern aus der Sprachanthropologie (bspw. Sprachideologien, Indexikalität), der Sprachpsychologie (bspw. Spracheinstellungen, Sprachperzeption) und der Sprachsoziologie (bspw. Identität und Alterität). 
de Konfliktgeschichte zwischen Sprachwissenschaft und sog. ,Öffentlichkeit ${ }^{20}$ deutlich gezeigt (vgl. Spitzmüller 2005a; 2005b). Für die derart sich verschmäht empfindenden Expert*innen selbst war dies zweifellos eine bittere Erfahrung (vgl. Antos, Tietz \& Weber 1999), die zu verschiedenen Verteidigungsstrategien geführt hat. Eine davon ist es, die Situation zu beklagen (vgl. bspw. Dieckmann 1991; Hoberg 1997). Häufig wird in diesem Zusammenhang darauf verwiesen, dass die Sprachwissenschaft aufgrund ihres Gegenstands in einer besonderen, also im Vergleich zu anderen Disziplinen prekäreren, Situation sei, weil - wie gern mit Verweis auf Goethe (Maximen und Reflexionen 1033; Goethe 2008 [1824]) angemerkt wird - „ein jeder, weil er spricht, glaubt, auch über Sprache sprechen zu können“ (vgl. etwa Hoberg in Wimmer et al. 1999: 265). ${ }^{21}$ Angesichts der (überwiegend von Naturwissenschaftler`innen geführten) Diskussionen unter dem Label Public Understanding of Science (vgl. Irwin \& Wynne 1998; Bucchi \& Trench 2008) und ganz analoger Diskussionen in anderen geistes- und kulturwissenschaftlichen Fächern (vgl. Luginbühl \& Schröter 2018) darf man diese selbst zugeschriebene Exklusivität durchaus anzweifeln und vermuten, dass auch dies vor allem der Selbstvergewisserung dient. Eine andere, häufig damit verbundene Strategie ist es, sich über nicht-linguistische Sprachwahrnehmungen zu mokieren, was wohl überwiegend im internen Kreis im Rahmen von Vorträgen geschieht. Hier werden dann Laien zu sprachreflexiven Ignoranten (vgl. Spitzmüller, Antos \& Niehr 2015: 316) oder Dilettanten (vgl. Schützeichel 2007: 547-548) degradiert. Beide Strategien sind aber angesichts zunehmender Legitimationsanforderungen unter den Fahnenwörtern Third Mission, Citizen Science, Critical Citizens und Selbstermächtigung (vgl. Bock \& Antos 2019; Claes 2005; Luginbühl \& Schröter 2018) wissenschaftspolitisch schwer durchzuhalten.

Ein anderes Verhältnis zu Laien suchen die Folk bzw. Perceptual Linguistics (vgl. Niedzielski \& Preston 2000; Preston 2019), die sprachpsychologische Spracheinstellungsforschung (vgl. Garrett 2010; Soukup 2019) und die sprachanthropologische Sprachideologieforschung (vgl. Kroskrity, Schieffelin \& Woolard 1998; Busch 2019). Sie sind mit dem Anspruch angetreten, zu verstehen, wie ,Laien' über Sprache denken bzw. reden. Dabei geht man explizit von der Annahme aus, dass es ,Laientheorien“ gibt, die ihrerseits konsistent sind, sich aber von sprachwissenschaftlichen Theorien signifikant unterscheiden können (vgl. bspw. Silverstein 1979: 193; Preston 2013: 62-64). Die Figur des

20 Vgl. kritisch zu dieser Gegenüberstellung Spitzmüller (2019).

21 Kritisch hierzu schon zwei Jahrzehnte zuvor von Polenz (1980: 31): „Ein jeder, weil er spricht, darf und soll auch über Sprache sprechen können“. 
Laien wird hier also deutlich anders konzipiert: Sie ist nicht nur Zuhörerin, ihr soll auch zugehört werden. Mit Blick auf Abbildung 2 könnte man sagen: aus dem Schalltrichter wird ein Sprechtrichter. ${ }^{22}$ Damit ist aber noch nicht gesagt, dass Laien damit mehr werden als Datenlieferanten, deren Stimmen kategorisiert und in den Kellerarchiven der Akademien katalogisiert werden. Wenn der Anspruch sich also darin erschöpft, ,Laienwissen' zu deuten und zu klassifizieren - um den Laien dann am Ende vielleicht noch, im Habitus Giddens', expertenhaft zu erklären, was es eigentlich ist, was sie ,wissen“ -, werden aus den Stimmen der ,Laien“ allenfalls gebändigte oder gemeisterte Stimmen (mastered voices im aufnahmetechnischen, im epistemologischen wie im machtprozeduralen Sinne). Die Frage ist, ob man der Figur des ,sprachlichen Laien“ damit gerecht wird - insbesondere dann, wenn man ,das' Laienwissen, wie es übrigens auch Preston (2013: 62-64) tut ${ }^{23}$, vor allem kontrastiv aus dem ,linguistischen Wissen' (was immer das in dieser Pauschalität sein soll) heraus entwickelt (unter Rückgriff auf Binarismen wie Deskription/Präskription, Rationalität/Emotion, Nähe/Distanz usw., die wohl mehr über Expertenpositionierungen erzählen als über Laienwissen).

Denn was über all diese wichtigen Anstrengungen zum Verständnis alltagsweltlicher Sprachreflexion hinaus nötig ist, wenn Laien, Wissen und Sprache in ein Verhältnis gesetzt werden sollen, ist, wie dieser Beitrag zu plausibilisieren versucht hat, eine Blickumkehr: ein reflexiver Blick auf die Figur und diskursive Position des Experten, die man nicht ausblenden kann, wenn man die Figur und diskursive Position(ierung) des Laien (vor allem die durch den Experten) verstehen will. Wer über Laien, Wissen und Sprache diskutieren will, sollte den Experten, sein ,Wissen“ und seine ,Sprache‘ also nicht vergessen.

22 Und wenn man den Einsatz von Phonographen in der Dialektologie, Phonologie und Anthropologie des späten 19. und frühen 20. Jahrhunderts bedenkt (vgl. https://www.oeaw.ac.at/ phonogrammarchiv/forschung/wissenschaftshistorische-forschung/ [letzter Zugriff 18.01.2020] sowie auch das Titelbild von R. Bauman \& Briggs 2003), ist dies nicht einmal nur metaphorisch zu verstehen.

23 „In the folk theory, just the opposite is true“ (Preston 2013: 64). 


\section{Literaturverzeichnis}

Agha, Asif (2007): The object called „language“ and the subject of linguistics. Journal of English Linguistics 35 (3), 217-235.

Althusser, Louis (1977 [1970]): Ideologie und ideologische Staatsapparate (Anmerkungen für eine Untersuchung). Übers. v. Rolf Löper, Peter Schöttler \& Klaus Riepe. In Ders., Ideologie und ideologische Staatsapparate: Aufsätze zur marxistischen Theorie, 108-153. Hamburg, Berlin: VSA. [Orig. frz.: Idéologie et appareils idéologiques d'état (Notes pour une recherche) . In La Pensée 151 (1970), 3-38].

Andersen, Christiane, Magnus P. Ängsal, Waldemar Czachur, Philipp Dreesen, Ulla Fix, Nina Kalwa, Jana Kiesendahl, Jürgen Schiewe, Jürgen Spitzmüller \& Barbara Zimmermann (2018): Erkenntnis als soziale Praxis: Ludwik Flecks Wissenschaftstheorie aus sprachwissenschaftlicher Sicht. In Christiane Andersen, Ulla Fix \& Jürgen Schiewe (Hrsg.), Denkstile in der deutschen Sprachwissenschaft: Bausteine einer Fachgeschichte aus dem Blickwinkel der Wissenschaftstheorie Ludwik Flecks (Philologische Studien und Quellen 265), 1165. Berlin: Erich Schmidt Verlag.

Anderwald, Lieselotte (Hrsg.) (2012): Sprachmythen - Fiktion oder Wirklichkeit. Frankfurt a. M. u. a. Peter Lang.

Antony, Alexander \& Michaela Pfadenhauer (2019): „Kritischer Journalismus unter Druck“: Zur Bedrohung und Verteidigung journalistischer Expertenkompetenz in der medialen Berichterstattung. In Nicole Burzan (Hrsg.), Komplexe Dynamiken globaler und lokaler Entwicklungen: Verhandlungen des 39. Kongresses der Deutschen Gesellschaft für Soziologie in Göttingen 2018. Essen: Deutsche Gesellschaft für Soziologie.

https://publikationen.soziologie.de/index.php/kongressband_2018/article/view/1024/ 1286 (letzter Zugriff 16.01.2020).

Antos, Gerd (1996): Laien-Linguistik: Studien zu Sprach- und Kommunikationsproblemen im Alltag. Am Beispiel von Sprachratgebern und Kommunikationstrainings (Reihe Germanistische Linguistik 146). Tübingen: Niemeyer.

Antos, Gerd, Heike Tietz \& Tilo Weber (1999): Linguistik in der Öffentlichkeit? Ergebnisse einer Umfrage unter LinguistInnen zum Forschungstransfer. In Gerhard Stickel (Hrsg.), Sprache - Sprachwissenschaft - Öffentlichkeit (Jahrbuch des Instituts für Deutsche Sprache 1998), 100-120. Berlin, New York: De Gruyter.

Bauman, Richard \& Charles L. Briggs (2003): Voices of modernity: Language ideologies and the politics of inequality (Studies in the Social and Cultural Foundations of Language 21). Cambridge: Cambridge University Press.

Bauman, Zygmunt (1987): Legislators and interpreters: On modernity, post-modernity and intellectuals. Cambridge: Polity Press.

Blommaert, Jan (2005): Discourse: A critical introduction (Key Topics in Sociolinguistics). Cambridge: Cambridge University Press.

Bock, Bettina \& Gerd Antos (2019): ,Öffentlichkeit‘ - ,Laien‘ - ,Experten‘: Strukturwandel von ,Laien“ und ,Experten` in Diskursen über ,Sprache‘. In Gerd Antos, Thomas Niehr \& Jürgen Spitzmüller (Hrsg.), Handbuch Sprache im Urteil der Öffentlichkeit (Handbücher Sprachwissen 10), 54-79. Berlin, Boston: De Gruyter.

Bogner, Alexander (2005): Grenzpolitik der Experten: Vom Umgang mit Ungewissheit und Nichtwissen in pränataler Diagnostik und Beratung. Braunschweig, Weilerswist: Velbrück Wissenschaft. 
Bucchi, Massimiano \& Brian Trench (Hrsg.) (2008): Handbook of public communication of science and technology. London: Routledge.

Busch, Brigitta (2019): Sprachreflexion und Diskurs: Theorien und Methoden der Sprachideologieforschung. In Gerd Antos, Thomas Niehr \& Jürgen Spitzmüller (Hrsg.), Handbuch Sprache im Urteil der Öffentlichkeit (Handbücher Sprachwissen 10), 107-139. Berlin, Boston: De Gruyter.

Cameron, Deborah (1995): Verbal hygiene (Language and Politics). London: Routledge.

Carr, E. Summerson (2010): Enactments of expertise. Annual Review of Anthropology 39, 1732.

Chomsky, Noam (2002): On nature and language. Cambridge: Cambridge University Press.

Claes, Tom (2005): Defining „the university“: From „ivory tower“ to „convenience store“. In Frank McMahon \& Tom Claes (Hrsg.), Probing the boundaries of higher education, 35-49. Oxford: Inter-Disciplinary Press.

Coupland, Nikolas (2001): Introduction: Sociolinguistic theory and social theory. In Nikolas Coupland, Srikant Sarangi \& Christopher N. Candlin (Hrsg.), Sociolinguistics and Social Theory (Language in Social Life Series), 1-26. London, New York: Routledge.

Denkler, Markus (Hrsg.) (2008): Frischwärts und unkaputtbar: Sprachverfall oder Sprachwandel im Deutschen. Münster: Aschendorff.

Dieckmann, Walther (1991): Sprachwissenschaft und öffentliche Sprachdiskussion - Wurzeln ihres problematischen Verhältnisses. In Rainer Wimmer (Hrsg.), Das 19. Jahrhundert: Sprachgeschichtliche Wurzeln des heutigen Deutsch (Jahrbuch des Instituts für Deutsche Sprache 1990), 355-371. Berlin, New York: De Gruyter.

Duranti, Alessandro (2006): Agency in language. In Alessandro Duranti (Hrsg.), A companion to Linguistic Anthropology (Blackwell Companions to Anthropology), 451-473. Oxford, Cambridge: Blackwell.

Fingerhuth, Matthias \& Hans Boas (2018): Anglizismen zwischen Linguistik und LaienLinguistik: Zum Fremdwortpurismus des Vereins Deutsche Sprache im Anglizismen-Index. Eine frame-semantische Analyse seiner Metatexte. In Csaba Földes (Hrsg.), Sprach- und Textkulturen - interkulturelle und vergleichende Konzepte (Beiträge zur Interkulturellen Germanistik 11), 19-41. Tübingen: Narr Francke Attempto.

Fleck, Ludwik (1999 [1935]): Entstehung und Entwicklung einer wissenschaftlichen Tatsache: Einführung in die Lehre vom Denkstil und Denkkollektiv. Mit einer Einl. v. Lothar Schäfer \& Thomas Schnelle. 4. Aufl. (stw 312). Frankfurt a. M.: Suhrkamp. [Orig.: Basel: Benno Schwabe].

Fleck, Ludwik (2011a [1936]): Das Problem einer Theorie des Erkennens. Übers. v. Bogustaw Wolniewicz \& Thomas Schnelle. In Sylwia Werner \& Claus Zittel (Hrsg.), Ludwik Fleck: Denkstile und Tatsachen. Gesammelte Schriften und Zeugnisse (stw 1953), 260-309. Frankfurt a. M.: Suhrkamp. [Orig. poln.: Zagadnienie teorii poznawania. Przeglad Filozoficzny 39 (1936), 3-37].

Fleck, Ludwik (2011b [1935]): Über die wissenschaftliche Beobachtung und die Wahrnehmung im Allgemeinen. Übers. v. Bogusław Wolniewicz \& Thomas Schnelle. In Sylwia Werner \& Claus Zittel (Hrsg.), Ludwik Fleck: Denkstile und Tatsachen. Gesammelte Schriften und Zeugnisse (stw 1953), 211-238. Frankfurt a. M.: Suhrkamp. [Orig. poln.: 0 obserwacji naukowej i postrzeganiu wogóle. Przegląd Filozoficzny 38 (1935), 58-76].

Foucault, Michel (1997 [1971]): Die Ordnung des Diskurses: Inauguralvorlesung am Collège de France, 2. Dezember 1970. Übers. v. Walter Seitter. Mit einem Vorw. v. Ralf Konersmann 
(Fischer Wissenschaft 10083). Frankfurt a. M.: Fischer. [Orig. frz.: L'ordre du discours : Leçon inaugurale au Collège de France prononcée le 2 décembre 1970. Paris: Gallimard].

Furnham, Adrian (1988): Lay Theories: Everyday Understanding of Problems in the Social Sciences. Oxford u. a.: Pergamon Press.

Garrett, Peter (2010): Attitudes to language (Key Topics in Sociolinguistics). Cambridge: Cambridge University Press.

Giddens, Anthony (1984): The constitution of society: Outline of the theory of structuration. Cambridge: Polity Press.

Giddens, Anthony (1987): What do sociologists do? In Ders., Social theory and modern sociology, 1-21. Stanford, CA: Stanford University Press.

Goethe, Johann Wolfgang von (2008 [1824]): Maximen und Reflexionen 1033. In Ders., Schriften zur Kunst. Schriften zur Literatur. Maximen und Reflexionen. Textkritisch durchges. v. Erich Trunz u. Hans Joachim Schrimpf (Goethes Werke. Hamburger Ausgabe in 14 Bänden, 12). 14. überarb. Aufl., 511. München: Beck.

Goffman, Erving (1959): The presentation of self in everyday life. New York: Doubleday.

Grewendorf, Günther (1993): Der Sprache auf der Spur: Anmerkungen zu einer Linguistik nach Jäger Art. Zeitschrift für Sprachwissenschaft 12 (1), 113-132.

Hall, Robert A. (1950): Leave your language alone! Ithaca, NY: Linguistica.

Hitzler, Ronald (Hrsg.) (1994): Expertenwissen: Die institutionalisierte Kompetenz zur Konstruktion von Wirklichkeit. Opladen: Westdeutscher Verlag.

Hoberg, Rudolf (1997): Öffentlichkeit und Sprachwissenschaft. Muttersprache 107 (1), 54-63.

Hoffmeister, Toke (2019): Laien als Experten und Experten als Laien: Zur Problematik eines etablierten Begriffspaares. Linguistik online 99 (6), 151-174. http://dx.doi.org/10.13092/lo.99.5969 (letzter Zugriff 16.01.2020).

Huber, Brigitte (2014): Öffentliche Experten: Über die Medienpräsenz von Fachleuten. Wiesbaden: Springer VS.

Irwin, Alan \& Brian Wynne (Hrsg.) (1998): Misunderstanding science? The public reconstruction of science and technology. Cambridge: Cambridge University Press.

Jäger, Ludwig (1993a): ,Language, whatever that may be‘: Die Geschichte der Sprachwissenschaft als Erosionsgeschichte ihres Gegenstandes. Zeitschrift für Sprachwissenschaft 12 (1), 77-106.

Jäger, Ludwig (1993b): „Chomsky’s problem“: Eine Antwort auf Bierwisch, Grewendorf und Habel. Zeitschrift für Sprachwissenschaft 12 (2), 235-260.

Joas, Hans \& Wolfgang Knöbl (2004): Sozialtheorie: Zwanzig einführende Vorlesungen (stw 1669). Frankfurt a. M.: Suhrkamp.

Kalverkämper, Hartwig (1990): Gemeinsprache und Fachsprachen: Plädoyer für eine integrierende Sichtweise. In Gerhard Stickel (Hrsg.), Deutsche Gegenwartssprache: Tendenzen und Perspektiven (Jahrbuch des Instituts für Deutsche Sprache 1989), 88-133. Berlin, New York: De Gruyter.

Kim, Kyung-Man (2004): Critical theory criticized: Giddens's double hermeneutic and the problem of language game change. Cultural Studies - Critical Methodologies 4 (1), 28-44.

Knobloch, Clemens (2005): Volkhafte Sprachforschung: Studien zum Umbau der Sprachwissenschaft in Deutschland zwischen 1918 und 1945 (Reihe Germanistische Linguistik 257). Tübingen: Niemeyer.

Knorr Cetina, Karin (1999): Epistemic cultures: How the sciences make knowledge. Cambridge, MA: Harvard University Press. 
Kroskrity, Paul V., Bambi B. Schieffelin \& Kathryn A. Woolard (Hrsg.) (1998): Language Ideologies: Practice and Theory (Oxford Studies in Anthropological Linguistics 16). New York: Oxford University Press.

Kuhn, Thomas S. (1999 [1962]): Die Struktur wissenschaftlicher Revolutionen. Übers. v. Hermann Vetter \& Kurt Simon. 15. Aufl. (stw 25). Frankfurt a. M.: Suhrkamp. [Orig.: The Structure of Scientific Revolutions. Chicago: University of Chicago Press].

Lobenstein-Reichmann, Anja (2013): Sprachliche Ausgrenzung im späten Mittelalter und der frühen Neuzeit (Studia Linguistica Germanica 117). Berlin, Boston: De Gruyter.

Longino, Helen (2016): The social dimensions of scientific knowledge. In Edward N. Zalta (Hrsg.), The Stanford encyclopedia of philosophy. Stanford: Stanford University. https://plato.stanford.edu/archives/spr2016/entries/scientific-knowledge-social (letzter Zugriff 27.09.2018).

Luginbühl, Martin \& Juliane Schröter (Hrsg.) (2018): Geisteswissenschaften und Öffentlichkeit: Linguistisch betrachtet (Sprache in Kommunikation und Medien 11). Frankfurt a. M. u. a.: Peter Lang.

Lyons, John (1968): Introduction to theoretical linguistics. Cambridge: Cambridge University Press.

Maas, Utz (2016): Sprachforschung in der Zeit des Nationalsozialismus: Verfolgung, Vertreibung, Politisierung und die inhaltliche Neuausrichtung der Sprachwissenschaft (Studia Linguistica Germanica 124). Berlin, Boston: De Gruyter.

Matoesian, Gregory M. (1999): The grammaticalization of participant roles in the constitution of expert identity. Language in Society 28, 491-521.

Meinunger, André (2008): Sick of Sick? Ein Streifzug durch die Sprache als Antwort auf den „Zwiebelfisch“. Berlin: Kulturverlag Kadmos.

Niedzielski, Nancy A. \& Dennis R. Preston (2000): Folk linguistics (Trends in Linguistics: Studies and Monographs 122). Berlin, New York: Mouton de Gruyter.

Nieto-Galan, Agusti (2016): Science in the public sphere: A history of lay knowledge and expertise. London, New York: Routledge.

Paul, Ingwer (1999): Praktische Sprachreflexion. In Brigitte Döring, Angelika Feine \& Wilhelm Schellenberg (Hrsg.), Über Sprachhandeln im Spannungsfeld von Reflektieren und Benennen (Sprache - System und Tätigkeit 28), 193-204. Frankfurt a. M. u. a.: Peter Lang.

Pfadenhauer, Michaela (2010): Der Experte. In Stephan Moebius (Hrsg.), Diven, Hacker, Spekulanten: Sozialfiguren der Gegenwart, 98-107. Berlin: Suhrkamp.

Polenz, Peter von (1980): Wie man über Sprache spricht: Über das Verhältnis zwischen wissenschaftlicher und natürlicher Beschreibungssprache in Sprachwissenschaft und Sprachlehre (Duden-Beiträge 5). Mannheim u. a.: Dudenverlag.

Polenz, Peter von (1988): Deutsche Satzsemantik: Grundbegriffe des Zwischen-den-ZeilenLesens. 2., durchges. Aufl. (Sammlung Göschen 2226). Berlin, New York: De Gruyter.

Popper, Karl R. (2005 [1935]): Logik der Forschung. 11. Aufl. Tübingen: Mohr. [Orig.: Wien: Springer].

Preston, Dennis R. (2013): Language with an attitude. In J. K. Chambers \& Natalie Schilling (Hrsg.), The handbook of language variation and change, 2. Aufl. (Blackwell Handbooks in Linguistics), 40-66. Oxford, Cambridge: Blackwell.

Preston, Dennis R. (2019): Folk linguistics and the perception of language variety. In Gerd Antos, Thomas Niehr \& Jürgen Spitzmüller (Hrsg.), Handbuch Sprache im Urteil der Öffentlichkeit (Handbücher Sprachwissen 10), 140-164. Berlin, Boston: De Gruyter. 
Quine, Willard Van Orman (1953 [1951]): Two dogmas of empiricism. In Ders., From a logical point of view: 9 logico-philosophical essays, 20-46. Cambridge, MA: Harvard University Press. [Zuerst in: The Philosophical Review 60 (1951), 20-43].

Russell, Bertrand (1910-1911): Knowledge by acquaintance and knowledge by description. Proceedings of the Aristotelian Society (New Series) 11. 108-128.

Sanders, Willy (1998): Sprachkritikastereien. 2., überarb. Aufl. Darmstadt: Wissenschaftliche Buchgesellschaft.

Schützeichel, Rainer (2007): Laien, Experten, Professionen. In Rainer Schützeichel (Hrsg.), Handbuch Wissenssoziologie und Wissensforschung, 546-578. Konstanz: UVK.

Silverstein, Michael (1979): Language structure and linguistic ideology. In Paul R. Cline, William Hanks \& Carol Hofbauer (Hrsg.), The elements: A parasession on linguistic units and levels, 193-247. Chicago: Chicago Linguistic Society.

Silverstein, Michael (2003): Indexical order and the dialectics of sociolinguistic life. Language \& Communication 23 (3-4), 193-229.

Soukup, Barbara (2019): Sprachreflexion und Kognition: Theorien und Methoden der Spracheinstellungsforschung. In Gerd Antos, Thomas Niehr \& Jürgen Spitzmüller (Hrsg.), Handbuch Sprache im Urteil der Öffentlichkeit (Handbücher Sprachwissen 10), 83-106. Berlin, Boston: De Gruyter.

Spitzmüller, Jürgen (2005a): Das Eigene, das Fremde und das Unbehagen an der Sprachkultur: Überlegungen zur Dynamik sprachideologischer Diskurse. Aptum: Zeitschrift für Sprachkritik und Sprachkultur 1 (3), 248-261.

Spitzmüller, Jürgen (2005b): Metasprachdiskurse: Einstellungen zu Anglizismen und ihre wissenschaftliche Rezeption (Linguistik - Impulse \& Tendenzen 11). Berlin, New York: De Gruyter.

Spitzmüller, Jürgen (2009): Sprachliches Wissen diesseits und jenseits der Linguistik. In Tilo Weber \& Gerd Antos (Hrsg.), Typen von Wissen: Begriffliche Unterscheidung und Ausprägungen in der Praxis des Wissenstransfers (Transferwissenschaften 7), 112-126. Frankfurt a. M. u. a.: Peter Lang.

Spitzmüller, Jürgen (2013): Metapragmatik, Indexikalität, soziale Registrierung: Zur diskursiven Konstruktion sprachideologischer Positionen. Zeitschrift für Diskursforschung 1 (3), 263-287.

Spitzmüller, Jürgen (2019): Sociolinguistics going ,wild': The construction of auratic fields. Journal of Sociolinguistics 23 (5), 505-520.

Spitzmüller, Jürgen, Gerd Antos \& Thomas Niehr (2015): Sprache im Urteil der Öffentlichkeit. In Ekkehard Felder \& Andreas Gardt (Hrsg.), Handbuch Sprache und Wissen (Handbücher Sprachwissen 1), 314-331. Berlin, Boston: De Gruyter.

Stegu, Martin (i. Dr.): Linguistes vs. non-linguistes: Rôle et statut des acteurs. Erscheint in Lidia Becker, Herling Sandra \& Holger Wochele (Hrsg.), Manuel de linguistique populaire (Manuals of Romance linguistics). Berlin, Boston: De Gruyter.

Strauß, Gerhard, Heidrun Kämper, Isolde Nortmeyer, Herbert Schmidt \& Oda Vietze (2011) : Deutsches Fremdwörterbuch. 2. Aufl., völlig neu bearb. im Institut für Deutsche Sprache. Bd. 5: Eau de Cologne - Futurismus. Berlin, New York: De Gruyter.

Suisman, David (2009): Selling sounds: The commercial revolution in American music. Cambridge, MA, London: Harvard University Press.

Wimmer, Rainer, Rudolf Hoberg, Fritz Kuhn, Eva Neuland, Achim Struchholz \& Annette Trabold (1999): Podiumsdiskussion: Was nun? Wie kann man das Verhältnis Sprachwissenschaft - Öffentlichkeit verbessern? In Gerhard Stickel (Hrsg.), Sprache - Sprachwissenschaft - 
Öffentlichkeit (Jahrbuch des Instituts für Deutsche Sprache 1998), 262-282. Berlin, New York: De Gruyter. 
